\title{
Commentaries
}

\section{Lost in transdifferentiation}

\author{
Mark H. Hoofnagle, Brian R. Wamhoff, and Gary K. Owens \\ Department of Molecular Physiology and Biological Physics, University of Virginia, Charlottesville, Virginia, USA.
}

\begin{abstract}
What are the true origins of the smooth muscle cells (SMCs) present in the intimal lesions of transplant arteriosclerosis? A new study in the JCI shows that $\mathrm{Sca}^{-1^{+}}$cells purified from the mouse aortic root can migrate through an irradiated vein graft to the neointima of the vessel and transdifferentiate to express the early SMC differentiation marker gene SM22 (see the related article beginning on page 1258 ). Do Sca- $1^{+}$cells transdifferentiate into SMClike cells, or is activation of SMC marker genes a consequence of fusion of these cells with preexisting SMCs, a possibility raised by results of studies of adult stem cells in animal models of liver regeneration (see the related article beginning on page 1266)? Or could this be bona fide transdifferentiation that recapitulates the pathologic processes in humans?
\end{abstract}

The prevailing theory of smooth muscle cell (SMC) contribution to vessel lesions is that in pathological states, such as injury and atherosclerosis, SMCs migrate to the intima from the media of the vessel (1). This theory, which has persisted for three decades, is now being challenged by results from models of vessel injury, transplant arteriosclerosis (TA) models, and human allograft studies indicating that a portion of the cells bearing SMC differentiation markers in intimal lesions may have originated from the hematopoietic system and/ or circulating progenitor cells (2-5). However, these studies show variable contributions of marrow-derived cells to lesions, with increased frequency correlated to severity of medial injury or to degree of donor/allograft mismatch (6). It appears that only with necrosis of medial SMCs are bone marrow cell (BMC) investment frequencies extremely high (7), indicating that the marrow is not solely responsible for populating the intimal lesion but may represent a default pathway for SMC regeneration and vessel repair in circumstances of severe vessel wall damage.

Two seminal studies by Hu et al. provide new insights into the origin of cells populating the developing neointima in a mouse model of TA $(8,9)$. Using a combination of bone marrow reconstitution and vein allografts (Figure 1), they demonstrated that donor BMCs give rise to neointimal

Nonstandard abbreviations used: bone marrow cell (BMC); hematopoietic stem cell (HSC); smooth muscle (SM); SM cell (SMC); transplant arteriosclerosis (TA).

Conflict of interest: The authors have declared that no conflict of interest exists.

Citation for this article: J. Clin. Invest. 113:1249-1251 (2004). doi:10.1172/JCI200421761. cells and smooth muscle (SM) $\alpha$-actin-positive cells $(2,3)$, although evidence for this is somewhat controversial due to the lack of high-resolution confocal microscopic analyses showing definitive colocalization of bone marrow lineage marker genes and SM $\alpha$-actin immunostaining (6). However, bone marrow reconstitution studies using donor bone marrow from SM22 promoter-LacZtransgenic mice have shown that donor BMCs do not give rise to SM22-expressing neointimal cells $(2,3)$ - a surprising result, since SM22 is an early SMC differentiation marker gene (6). Donor vein graft cells also do not contribute SM22-positive neointimal cells, but host vessel-derived cells can give rise to the population of SM22-positive graft neointimal cells. However, it must be noted that the failure of donor vein graft cells to contribute to the intima appears to be unique to this allograft model, wherein there is virtual destruction of all donor vessel cells, including medial SMCs. Thus, neointimal cells that express SM22 do not appear to be bone marrow-derived but rather to have some other host-based source.

In this issue of the JCI, Hu and colleagues hypothesize that in addition to circulating progenitor cells, Sca- $1^{+}$progenitor cells that reside in the adventitia may transdifferentiate into SMC-like neointimal cells (10). These results provide fresh arguments in a key controversy in adult stem cell and progenitor cell research -i.e., that of defining the relative roles of cell fusion versus transdifferentiation.

The role of adult stem cell contribution to tissues has recently undergone a revolution and counterrevolution. Initial optimism about transdifferentiation of neuronal stem cells (11), hematopoietic stem cells
(HSCs) (12), and other adult stem cell types has diminished in the face of reports of cell fusion (13) and minimal adult stem cell plasticity (14). Surprisingly, the pair of papers that began the furor over cell fusion throughout the adult stem cell field had little relevance to most cell types being studied. Terada et al. and Ying et al. demonstrated rare cell fusion events when embryonic stem cells are extensively cocultured with BMCs or neural cells, respectively $(15,16)$. These results, however, did lead to a healthy skepticism about the transdifferentiation potential of adult stem cells. Ianus et al. developed a Cre-lox model to specifically test for fusion in their cell systems, and found none, despite the apparent transdifferentiation of BMCs into insulin-secreting pancreatic $\beta$ cells (17). Some groups examining BMC transdifferentiation into hepatocytes found that their previous results were mainly due to cell fusion (13). Still others, alerted to the fusion problem, found cell fusion occurred in their systems, but at too low a frequency to account for their transdifferentiation results $(18,19)$.

Thus, a confusing picture has emerged for most scientists curious about the role of adult stem cells in human disease. We are left with several questions: Is transdifferentiation real or just an artifact? Is fusion a problem or is it a potential physiological mechanism to exploit? Are some tissues more susceptible to fusion than others? The liver, for instance, has proven to be highly susceptible to fusion, but, interestingly, in the fumarylacetoacetate bydrolase ${ }^{-1}$ mouse models studied, fusion of HSCs to hepatocytes corrects the enzymatic deficiency, raising the possibility that this phenomenon has therapeutic applications (12). Of note in this issue of the JCI is the work of Camargo et al., who explored the fusibility of hepatocytes with HSCs and discovered that it is specifically the myeloid lineage of these cells, rather than the stem cells themselves, that is responsible for fusion, thus raising the intriguing possibility of "fusion therapy" (20). These groundbreaking new results also emphasize the importance of understanding fusion and transdifferentiation, as they appear to play a potentially important role in several dis- 


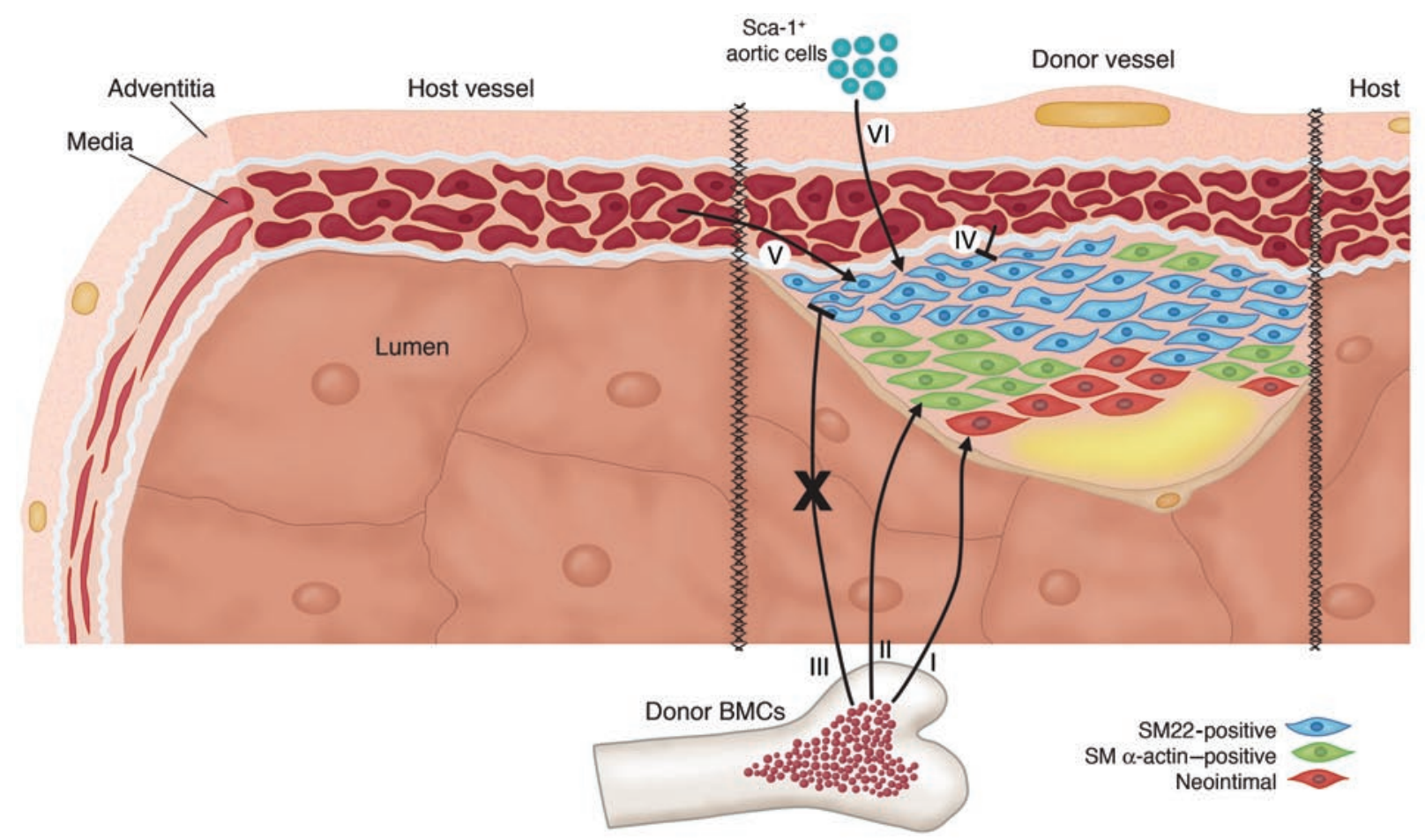

Figure 1

What are the true origins of the SMCs present in the intimal lesions of TA? Hu et al. previously demonstrated that donor BMCs give rise to neointimal cells (I) and that a low percentage costained with smooth muscle $\alpha$-actin, suggesting transdifferentiation of BMCs to SMCs. (II) However, when SM22-LacZ bone marrow was used for lineage tracing, the neointimal cells were SM22-LacZ negative, suggesting that the BMC-derived SM $\alpha$-actin-positive intimal cells were some cell type other than smooth muscle (e.g., macrophages or myofibroblasts) or were not fully differentiated SMCs (III). Interestingly, cells from the host vessel (V) but not the donor vessel (IV) can give rise to intimal cells that are SM22-positive. However, it must be noted that the failure of donor vessel cells to contribute to the intima appears to be unique to this allograft model, wherein there is virtual destruction of all donor vessel cells, including medial SMCs. Thus, in this allograft model, a key question is, what is the source of the SM22-positive intimal cells derived from the host vessel? In this issue of the $\mathrm{JCl}$, Hu et al. show that Sca- $1^{+}$cells purified from the adventitia of the aortic root $(\mathrm{VI})$ can migrate through an irradiated vein graft to the neointima of the vessel and express SM22. These results are of interest in that they identify a potential new source of cells that contribute to neointimal formation in TA. However, several outstanding questions remain: Is this an artifact of exogenous application of the Sca-1+ cells? Does it represent fusion of these cells to SMCs? Or does this represent bona fide transdifferentiation of Sca-1+ cells from the adventitia into SMC lineages?

ease states that biomedical scientists have been trying to understand for decades.

Adding to the controversy over the true transdifferentiation potential of adult stem cells are the exaggerated claims made by both the scientific and the lay press regarding the relative merits of adult versus embryonic stem cells and their potential for therapeutic applications. Both avenues of research would be better served by more accurate portrayals of their science in the press, which might help to lessen the impact of partisan politics on serious consideration of the merits of either stem cell type and their potential contributions to science and human therapeutics.

In this issue of the JCI, Hu et al. provide novel evidence that may result in a more coherent picture of different cell contributions to the lesions of TA. Of major interest is the fact that Hu et al. isolated a puta- tive SMC progenitor population from the adventitia of the aortic root with a surface phenotype of Sca- $1^{+} / \mathrm{c}-\mathrm{kit}^{+} / \mathrm{lin}^{-}$characteristic of HSCs. Further, through a series of lineage tracing studies, they demonstrated that these cells were not of hematopoietic origin, suggesting they are not HSCs that have lodged or fused in the adventitia. In culture, PDGF-BB induces expression of multiple markers of SMC differentiation, and these cells, when purified from an SM22-LacZ/ApoE $E^{-/-}$transgenic mouse and applied to the outside of an irradiated vein allograft, migrate to the neointima of the vessel and appear to activate de novo SM22 transcription in the neointima (Figure 1).

These results are exciting and significant in that they identify a potential new origin of cells that contribute to neointimal formation in TA. However, caution must be taken, as critical experiments are required to identify the physiological relevance of these cells and eliminate two limitations of this study. First, application of exogenous Sca- $1^{+}$cells to an irradiated vein graft represents an artificial situation in which migration may have been an experimental artifact due to lack of medial SMC contribution. To determine if such migration is physiologically relevant, a Sca-1 promoterCre mouse could be crossed to a ROSAstoplox-LacZ/ApoE $E^{-/-}$mouse to determine if Sca-1 was ever activated in neointimal SMCs. Second, fusion of Sca- $1^{+}$cells with cells from another source, such as irradiated SMCs of the media, must be eliminated, although other groups suggest that this is an unlikely eventuality (19). A Cre-lox fusion detection system (17) could be used to quell concerns that $\mathrm{Sca}-1^{+}$cells adopt the SMC phenotype by fusing to other SMCs rather than by transdifferentiating. 
From all studies so far we are still left with the fundamental question: Do non-SMCs from the adventitia, host vessel, or bone marrow transdifferentiate to become bona fide SMCs? It is possible that these cells are expressing only a few SMC differentiation markers and/or still expressing non-SMC genes. Finally, and most importantly, we ask, are the results in these animal models relevant to the development of atherosclerosis in humans? Can these same cells be identified in humans by Sca-1 antigen? The novel and exciting studies by Hu et al. in this issue of the JCI implicate potential contributions by a putative adventitial progenitor cell population in TA and might open yet another chapter in the long quest to define origins of cells in intimal lesions and their mechanistic contributions to the pathogenesis of atherosclerosis.

Mark H. Hoofnagle and Brian R. Wamhoff contributed equally to this work.

Address correspondence to: Gary K. Owens, Department of Molecular Physiology and Biological Physics, University of Virginia, MR5 Room 1220, 415 Lane Road, PO Box 801394, Charlottesville, Virginia 22908,
USA. Phone: (434) 924-2652; Fax: (434) 982-0055; E-mail: gko@virginia.edu.

1. Ross, R., and Glomset, J.A. 1973. Atherosclerosis and the arterial smooth muscle cell: proliferation of smooth muscle is a key event in the genesis of the lesions of atherosclerosis. Science. 180:1332-1339.

2. Sata, M., et al. 2002. Hematopoietic stem cells differentiate into vascular cells that participate in the pathogenesis of atherosclerosis. Nat. Med. 8:403-409.

3. Shimizu, K., et al. 2001. Host bone-marrow cells are a source of donor intimal smooth-muscle-like cells in murine aortic transplant arteriopathy. Nat. Med. 7:738-741.

4. Glaser, R., Lu, M.M., Narula, N., and Epstein, J.A. 2002. Smooth muscle cells, but not myocytes, of host origin in transplanted human hearts. Circula tion. 106:17-19.

5. Simper, D., Stalboerger, P.G., Panetta, C.J., Wang, S., and Caplice, N.M. 2002. Smooth muscle progenitor cells in human blood. Circulation. 106:1199-1204.

6. Owens, G.K., Kumar, M.S., and Wamhoff, B.R. 2004. Molecular regulation of vascular smooth muscle cell differentiation in development and disease. Physiol. Rev. In press.

7. Campbell, J.H., Han, C.L., and Campbell, G.R. 2001. Neointimal formation by circulating bone marrow cells. Ann. N. Y. Acad. Sci. 947:18-24.

8. Hu, Y., et al. 2002. Both donor and recipient origins of smooth muscle cells in vein graft atherosclerotic lesions. Circ. Res. 91:13e-20e.

9. $\mathrm{Hu}, \mathrm{Y}$., et al. 2002. Smooth muscle cells in transplant atherosclerotic lesions are originated from recipients, but not bone marrow progenitor cells. Circulation. 106:1834-1839.

10. Hu, Y., et al. 2004. Abundant progenitor cells in the adventitia contribute to atherosclerosis of vein grafts in ApoE-deficient mice. J. Clin. Invest. 113:1258-1265. doi:10.1172/JCI200419628.

11. Bjornson, C.R., Rietze, R.L., Reynolds, B.A., Magli, M.C., and Vescovi, A.L. 1999. Turning brain into blood: a hematopoietic fate adopted by adult neural stem cells in vivo. Science. 283:534-537.

12. Lagasse, E., et al. 2000. Purified hematopoietic stem cells can differentiate into hepatocytes in vivo. Nat. Med. 6:1229-1234.

13. Wang, X., et al. 2003. Cell fusion is the principal source of bone-marrow-derived hepatocytes. Nature. 422:897-901.

14. Wagers, A.J., Sherwood, R.I., Christensen, J.L., and Weissman, I.L. 2002. Little evidence for developmental plasticity of adult hematopoietic stem cells. Science. 297:2256-2259.

15. Terada, N., et al. 2002. Bone marrow cells adopt the phenotype of other cells by spontaneous cell fusion. Nature. 416:542-545

16. Ying, Q.L., Nichols, J., Evans, E.P., and Smith, A.G. 2002. Changing potency by spontaneous fusion. Nature. 416:545-548.

17. Ianus, A., Holz, G.G., Theise, N.D., and Hussain, M.A. 2003. In vivo derivation of glucose-competent pancreatic endocrine cells from bone marrow without evidence of cell fusion. J. Clin. Invest. 111:843-850. doi:10.1172/JCI200316502.

18. Spees, J.L., et al. 2003. Differentiation, cell fusion, and nuclear fusion during ex vivo repair of epithelium by human adult stem cells from bone marrow stroma. Proc. Natl. Acad. Sci. U. S. A. 100:2397-2402.

19. Saiura, A., et al. 2003. Little evidence for cell fusion between recipient and donor-derived cells. J. Surg. Res. 113:222-227.

20. Camargo, F.D., Finegold, M., and Goodell, M.A. 2004. Hematopoietic myelomonocytic cells are the major source of hepatocyte fusion partners. J. Clin. Invest. 113:1266-1270. doi:10.1172/JCI200421301.

\title{
Anemia of inflammation: the cytokine-hepcidin link
}

\author{
Nancy C. Andrews \\ Children's Hospital, Howard Hughes Medical Institute, Harvard Medical School, and Dana-Farber Cancer Institute, Boston, Massachusetts, USA.
}

\begin{abstract}
The anemia of inflammation, commonly observed in patients with chronic infections, malignancy, trauma, and inflammatory disorders, is a well-known clinical entity. Until recently, we understood little about its pathogenesis. It now appears that the inflammatory cytokine IL-6 induces production of hepcidin, an iron-regulatory hormone that may be responsible for most or all of the features of this disorder (see the related article beginning on page 1271).
\end{abstract}

In 1932, Locke et al. made the important observation that infection was associated with hypoferremia (low serum iron), providing a partial explanation for the common finding of anemia in patients with chronic infections (1). Cartwright and Wintrobe went on to show that the anemia associated with infection was indistinguishable from the anemia of inflam-

Conflict of interest: The author has declared that no conflict of interest exists.

Citation for this article: J. Clin. Invest. 113:1251-1253 (2004). doi:10.1172/JCI200421441. mation, and established that hypoferremia resulted from reticuloendothelial sequestration of iron and interruption of intestinal iron absorption $(2,3)$. Cartwright and Lee recognized that similar findings could be induced in mice by exposure to bacterial endotoxin (4). Others correlated the anemia of inflammation with elaboration of inflammatory cytokines, and ascribed changes in iron metabolism to the effects of these cytokines (5). Cytokines have been shown to modulate the expression of iron transport and storage proteins (6), but it was not clear that these changes accounted for the abnormalities of iron homeostasis observed in the anemia of inflammation.

\section{The roles of hepcidin and IL- 6}

Over the past two years, a variety of experiments have converged to establish a role for hepcidin, a liver-derived peptide regulator of iron homeostasis, as a key mediator of hypoferremia in inflammation (7-9). In an elegant report in this issue of the JCI, Nemeth, Rivera, and colleagues have elucidated an important link between inflammatory cytokines and hepcidin (10). Using both mice and humans as experimental models, they have shown that IL-6 acts directly on hepatocytes to stimulate hepcidin production. Hepcidin, in turn, acts as a negative regulator of intestinal iron absorption and macrophage iron release.

In previous work Nemeth, Rivera, and colleagues showed that IL-6 induced hepcidin expression in hepatic cells (9). 


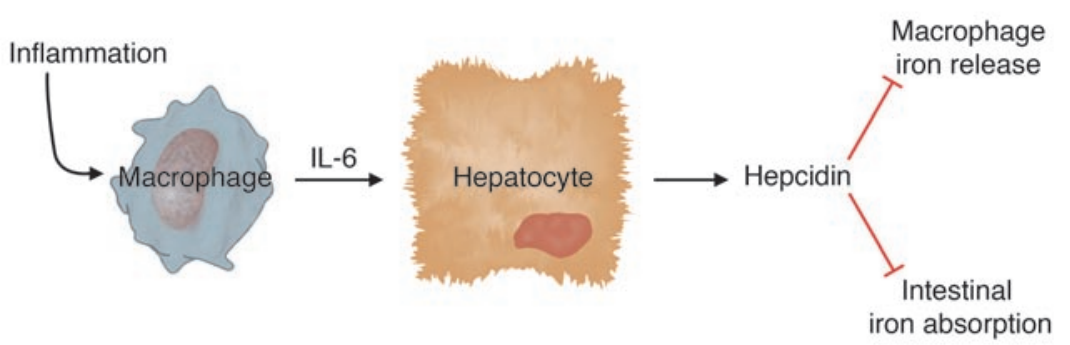

Here, they have replicated this effect using conditioned medium from endotoxintreated macrophages and shown that a neutralizing antibody against IL-6 blocked hepcidin induction (10). Other inflammatory cytokines did not stimulate hepcidin production; in fact, TNF- $\alpha$ inhibited it.

Cytokines have all sorts of effects on cultured cells, and it was important to show that IL-6 induction of hepcidin occurred in vivo and triggered hypoferremia, as predicted. First, Nemeth, Rivera, et al. used turpentine injection to cause inflammatory abscesses in wild-type and IL- 6 knockout mice and analyzed the responses (10). Wildtype mice had increased hepcidin expression and a substantial decrease in serum iron levels. In contrast, IL-6 knockout mice had no increase in hepcidin expression and no decrease in serum iron. A complementary experiment carried out in human volunteers showed that IL- 6 infusion stimulated urinary hepcidin excretion within 2 hours and induced hypoferremia. Taken together, these data provide strong support for the conclusions that IL- 6 is a primary inducer of hepcidin expression and that increased hepcidin expression results in hypoferremia (Figure 1). This is gratifyingly consistent with clinical observations that hypoferremia occurs very quickly after the onset of inflammation.

Earlier studies had shown that rodents with induced iron overload also had increased hepcidin expression $(11,12)$, presumably to try to compensate for iron excess. However, the signal to increase hepcidin expression was unknown. Here, Nemeth, Rivera, and colleagues have shown that IL-6 is not involved in the regulation of hepcidin in response to iron (10). Furthermore, their data suggest that hepcidin levels are not simply responding to increased iron stores. In human volunteers, urinary hepcidin levels were boosted soon after a single dose of oral iron, which should have no significant effect on iron stores. Perhaps the serum iron level, known to increase transiently after iron inges- tion, might itself be the signal to induce hepcidin expression. Alternatively, the signal might relate to the degree of iron saturation of serum transferrin.

However, if transferrin iron saturation modulates hepcidin expression, other signals can clearly override its effects. The IL-6-mediated inflammatory induction of hepcidin does not appear to be offset by the hypoferremia it causes, at least in the short term. And mice with thalassemia intermedia (which presumably have elevated serum iron) have decreased hepcidin expression (13), as occurs in other mouse models with increased erythroid iron demand $(7,8)$.

In my opinion, this report from Nemeth, Rivera, et al. (10) leaves little room for doubt about the importance of hepcidin in the pathogenesis of anemia of inflammation. This was challenged by a recent report that concluded that elevated serum hepcidin levels were not useful in the diagnosis of the anemia of inflammation (14). However, that study did not provide data to support the authors' contention that they had developed a sensitive, specific test for serum hepcidin. Furthermore, as they also pointed out, it was not clear that serum measurements were as useful as urinary hepcidin measurements. Hepcidin gene expression seems to be exquisitely sensitive to regulation, and the circulating peptide is small enough to be quantitatively filtered by the kidneys. Urine samples probably provide a better indication of recent hepcidin expression than individual serum samples.

\section{A possible treatment?}

If inflammatory induction of hepcidin causes hypoferremia, it is logical to predict that inhibition of hepcidin expression or activity would ameliorate the anemia of inflammation. Would that be advantageous? Perhaps, particularly in noninfectious inflammatory disorders. We know that patients (15) and mice (16) lacking hepcidin have increased intestinal iron absorption and increased serum iron, but

\section{Figure 1}

Regulation of hepcidin production in inflammation. Inflammation leads to macrophage elaboration of IL-6, which acts on hepatocytes to induce hepcidin production. Hepcidin inhibits macrophage iron release and intestinal iron absorption, leading to hypoferremia.

this is unlikely to be harmful in the short term. However, there may be more cause for concern in patients with infections or malignancy. Decreased serum iron is believed to contribute to host defense against invading pathogens and tumor cells (17), and hepcidin itself has antimicrobial properties of uncertain importance (18). If hepcidin antagonists become available, careful clinical trials will be required to define appropriate indications for their use.

Address correspondence to: Nancy C. Andrews, Children's Hospital, Howard Hughes Medical Institute, and Harvard Medical School, 300 Longwood Avenue, Boston, Massachusetts 02115-5737, USA. Phone: (617) 919-2116; Fax: (617) 730-0934; E-mail: nandrews@enders.tch.harvard.edu.

1. Locke, A., Main, E.R., and Rosbach, D.O. 1932. The copper and non-hemoglobinous iron contents of the blood serum in disease. J. Clin. Invest. 11:527-542.

2. Cartwright, G.E., and Wintrobe, M.M. 1952. The anemia of infection. XVII. A review. Adv. Intern. Med. 5:165-226.

3. Cartwright, G.E. 1966. The anemia of chronic disorders. Semin. Hematol. 3:351-375.

4. Cartwright, G.E., and Lee, G.R. 1971. The anaemia of chronic disorders. Br. J. Haematol. 21:147-152.

5. Means, R.T. 1995. Pathogenesis of the anemia of chronic disease: a cytokine-mediated anemia. Stem Cells. 13:32-37.

6. Ludwiczek, S., Aigner, E., Theurl, I., and Weiss, G. 2003. Cytokine-mediated regulation of iron transport in human monocytic cells. Blood. 101:4148-4154.

7. Weinstein, D.A., et al. 2002. Inappropriate expression of hepcidin is associated with iron refractory anemia: implications for the anemia of chronic disease. Blood. 100:3776-3781.

8. Nicolas, G., et al. 2002. The gene encoding the iron regulatory peptide hepcidin is regulated by anemia, hypoxia, and inflammation. J. Clin. Invest. 110:1037-1044. doi:10.1172/JCI200215686.

9. Nemeth, E., et al. 2003. Hepcidin, a putative mediator of anemia of inflammation, is a type II acutephase protein. Blood. 101:2461-2463.

10. Nemeth, E., et al. 2004. IL-6 mediates hypoferremia of inflammation by inducing the synthesis of the iron regulatory hormone hepcidin. J. Clin. Invest. 113:1271-1276. doi:10.1172/JCI200420945.

11. Pigeon, C., et al. 2001. A new mouse liver-specific gene, encoding a protein homologous to human antimicrobial peptide hepcidin, is overexpressed during iron overload. J. Biol. Chem. 276:7811-7819.

12. Muckenthaler, M., et al. 2003. Regulatory defects 
in liver and intestine implicate abnormal hepcidin and Cybrd 1 expression in mouse hemochromatosis. Nat. Genet. 34:102-107.

13. Adamsky, K., et al. 2004. Decreased hepcidin mRNA expression in thalassemic mice. Br.J. Haematol. 124:123-124.

14. Dallalio, G., Fleury, T., and Means, R.T. 2003.
Serum hepcidin in clinical specimens. Br. J. Haematol. 122:996-1000.

15. Roetto, A., et al. 2003. Mutant antimicrobial peptide hepcidin is associated with severe juvenile hemochromatosis. Nat. Genet. 33:21-22.

16. Nicolas, G., et al. 2001. Lack of hepcidin gene expression and severe tissue iron overload in upstream stimulatory factor 2 (USF2) knockout mice. Proc. Natl. Acad. Sci. U. S. A. 98:8780-8785.

17. Weinberg, E.D. 1986. Iron, infection and neoplasia. Clin. Physiol. Biochem. 4:50-60.

18. Park, C.H., Valore, E.V., Waring, A.J., and Ganz, T. 2001. Hepcidin, a urinary antimicrobial peptide synthesized in the liver. J. Biol. Chem. 276:7806-7810.

\title{
Hold the antioxidants and improve plasma lipids?
}

\author{
Ronald M. Krauss
}

Children's Hospital Oakland Research Institute, Oakland, California, USA.

\begin{abstract}
Intrahepatic proteolysis is a major determinant of secretion of ApoB-containing lipoproteins into plasma. Stimulation of post-ER presecretory proteolysis (PERPP) of ApoB by n-3 polyunsaturated fatty acids has been found to result in reduced secretion of VLDL particles by hepatocytes. A new study has shown that this stimulation is promoted by pro-oxidant conditions that result in increased hepatic lipid hydroperoxide content (see the related article beginning on page 1277). Conversely, PERPP is suppressed by antioxidants and by saturated fatty acids, which are not susceptible to lipid peroxidation. Hence reduction of oxidative stress may have the unexpected side effect of increasing plasma lipid levels.
\end{abstract}

Dietary fats with differing fatty acid composition can influence plasma lipid levels by modulating hepatic production and clearance of lipoproteins (1), as well as by altering activity of cholesteryl ester transfer protein (2). Suppression and stimulation of hepatic LDL receptor activity are major determinants, respectively, of the effects of saturated and polyunsaturated fatty acids on plasma LDL clearance (1), but the mechanisms for effects of specific fatty acids on hepatic lipoprotein production are less well understood. This is in large part due to the multiple influences of fatty acids on processes that regulate hepatic lipid production and storage, and processing of $\mathrm{ApoB}$ in conjunction with lipoprotein synthesis and secretion (3).

Fatty acids are critically involved in hepatic lipoprotein production pathways that help maintain hepatic cholesterol homeostasis and the ability to respond to energy and other metabolic needs. Triglycerides influence a critical early step in secretion of ApoB-containing lipoproteins, namely the cotranslational binding of lipids to ApoB in the ER mediated by micro-

Nonstandard abbreviations used: ER-associated degradation (ERAD); microsomal triglyceride transfer protein (MTP); post-ER presecretory proteolysis (PERPP); thiobarbituric acid-reactive substance (TBARS).

Conflict of interest: The author has declared that no conflict of interest exists.

Citation for this article: J. Clin. Invest. 113:1253-1255 (2004). doi:10.1172/JCI200421637. somal triglyceride transfer protein (MTP). The resulting protection of specific ApoB domains from proteolysis, termed ER-associated degradation (ERAD), leads either to secretion of a relatively lipid-depleted particle or to further, posttranslational lipidation (3) (Figure 1). The latter may occur in a graded manner in the ER, the vesicular tubular complex, and/or the Golgi apparatus, or by fusion with a preformed lipid droplet in the ER by a process that is not dependent on MTP.

\section{Post-ER presecretory proteolysis and} hepatic lipid hydroperoxide content

Recently, Fisher et al. have identified another degradative process that can modulate hepatic secretion of more mature ApoB-containing lipoproteins (4). They have found that inhibition of hepatic ApoB secretion by $n-3$ polyunsaturated fatty acids occurs via activation of this process, which has been designated postER presecretory proteolysis (PERPP) (4). In this issue of the JCI, Pan, Fisher, and colleagues have now shown, using several lines of evidence, that this effect is mediated by fatty acid peroxidation, and that it also occurs with n-6 polyunsaturated fatty acids (5). Moreover, the finding that ApoB degradation is stimulated by pro-oxidant conditions and inhibited by antioxidants raises the question of whether variation in intrahepatic oxidative stress contributes to physiologic and/or pathologic modulation of ApoB-containing lipoprotein metabolism. There is indeed abundant evidence that consumption of supplements of longer-chain n-3 marine-derived polyunsaturated fatty acids (eicosapentaenoic and docosahexanoic acids) can lower plasma triglyceride and VLDL levels in humans (6). Recently, it has been shown that population variation in dietary intake of a plantderived n-3 polyunsaturated fatty acid, linolenic acid, is significantly associated with plasma triglyceride levels, independent of other nutrients, including longerchain n-3 polyunsaturated fatty acids (7). As Pan et al. point out, however, in their study linolenic acid suppressed ApoB secretion from rat hepatoma cells to a greater extent than could be accounted for by the relation of secretion to intrahepatic lipid hydroperoxide content as assessed by levels of thiobarbituric acid-reactive substances (TBARSs) (5). It should be noted, however, that TBARSs do not represent the full spectrum of products of lipid peroxidation, such as F2-isoprostanes. Moreover, despite the capacity for peroxidation of both n-3 and n-6 polyunsaturated fatty acids, the latter have minimal and generally nonsignificant effects on plasma triglyceride levels in humans (8). Hence increased lipid peroxidation of n-3 fatty acids does not fully explain the effects of these fatty acids on hepatic ApoB-containing lipoprotein secretion. Other effects, such as reduced lipid synthesis, may also play a role.

\section{Oxidative stress and modulation of pathways for hepatic lipoprotein secretion}

The direct relation of the number of unsaturated bonds to lipid peroxidation is consistent with the lack of a suppressive effect of saturated fatty acids on plasma triglyceride levels. In contrast, a saturated fatty acid, myristic acid, was found to stimulate secretion of ApoB-containing 


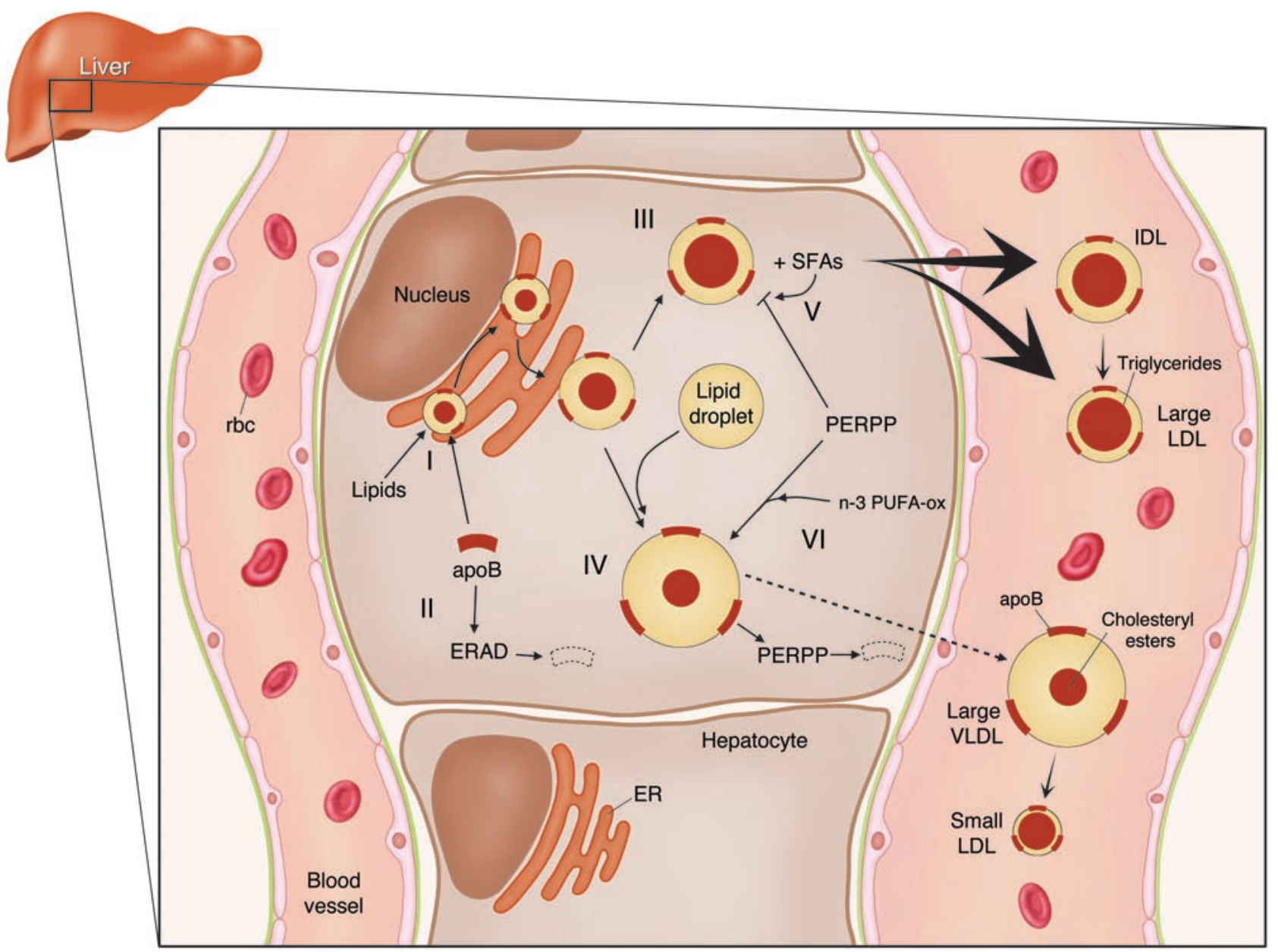

Figure 1

Scheme for the roles of intracellular proteolytic processes in regulating pathways for hepatic secretion of ApoB-containing lipoproteins and the modulating effects of saturated and n-3 fatty acids on these processes. Cotranslational lipidation of ApoB protects nascent particles from ERAD. Maturing particles can acquire choleteryl ester and be secreted as IDLs and larger LDLs or can fuse with a preformed lipid droplet to form larger VLDLs. Saturated fatty acids (SFAs) protect the smaller particles from PERPP, leading to increased secretion. Peroxidation products of $n-3$ polyunsaturated fatty acids (n-3 PUFA-ox), and perhaps also n-6 polyunsaturated fatty acids (not shown), increase PERPP, leading to decreased secretion of larger VLDLs and hence lower levels of their catabolic products, including small LDL particles.

lipoproteins from liver cells in conjunction with protection from a proteolytic process consistent with PERPP (9). Interestingly, the secreted lipoproteins under these conditions were denser than VLDLs and were more triglyceride-depleted than those secreted in the presence of albumin or oleic acid. This suggests that differences in conformation or composition of newly synthesized ApoB-containing lipoproteins can alter their exposure to PERPP in such a way that smaller, triglyceride-depleted particles formed under the influence of saturated fatty acids tend to escape this process, while larger, triglyceride-rich VLDLs are more susceptible. However, the results of Pan et al. (5) indicate that the stimulation of lipoprotein secretion by myristic acid is disproportionately greater than would be predicted by reduced hepatic TBARS content, suggesting, as was the case with linolenic acid, that effects other than those related to lipid peroxidation are involved.

Many questions remain as to the nature and physiologic importance of the PERPP system, the properties of lipoproteins that affect their susceptibility to PERPP, the mechanisms by which dietary fatty acids can affect this susceptibility, and the means by which oxidation products can suppress PERPP. It will be particularly challenging to assess the extent to which these mechanisms may influence metabolism of ApoBcontaining lipoproteins in humans. However, clues may be found in evidence that levels and distribution of LDL subspecies can serve as markers for intrahepatic pathways that result in formation of different hepatic secretory products (10) (Figure 1).

We have found that increased intake of saturated fatty acids is associated with higher levels of larger, more buoyant LDL particles (11). The concentrations of large, buoyant LDL particles are inversely related to plasma VLDL levels (12). Lipoprotein kinetic studies in humans have indicated that reduced plasma triglyceride and VLDL levels are correlated with the extent of direct secretion of IDLs and LDLs into plasma (13). Hence, one might surmise (Figure 1) that increased intake of cholesterol-raising saturated fatty acids increases direct hepatic secretion of IDLs and large LDLs, a process consistent with the evi- 
dence cited above that such secretion may be facilitated by protection from PERPP. Similarly, increased intake of $\omega-3$ fatty acids has been shown to result in a shift from small, dense LDLs to large, buoyant LDLs (14). This effect is likely to be multifactorial, with an important role for reduced cholesteryl ester transfer proteinmediated transfer of triglyceride to LDLs and subsequent lipolysis $(10,13)$. However, it is also consistent with evidence that larger, triglyceride-rich VLDLs are metabolic precursors of smaller LDL particles (10), and with the findings of Pan et al. (5) that increased PERPP reduces hepatic output of particles in this pathway (Figure 1).

A possible role for oxidation products in modulating hepatic lipoprotein secretion in humans can be assessed by determination of whether antioxidant treatment increases plasma transport or concentrations of ApoB-containing lipoproteins. In a randomized placebo-controlled study of 20,536 adults with pre-existing vascular disease or diabetes, daily supplementation with $600 \mathrm{mg}$ vitamin E, $250 \mathrm{mg}$ vitamin C, and $20 \mathrm{mg} \beta$-carotene resulted in small but statistically significant increases in triglyceride (11\%), ApoB (5\%), and LDL cholesterol (3\%), along with a small reduction in HDL cholesterol (15). No changes in triglyceride or LDL levels following antioxidant supplementation were found in a much smaller trial, but there was a reduction in the HDL2 cholesterol fraction, which was associated with reduced benefit on coronary disease endpoints when antioxidants were combined with simvastatin plus niacin therapy (16). Thus, although there is considerable evidence for the involvement of oxidative stress in many disease processes, including atherosclerosis, the potential for unintended outcomes of antioxidant therapy should serve as a warning against proceeding with such treatment in the absence of clinicaltrial evidence for benefit and safety.

Address correspondence to: Ronald M. Krauss, Children's Hospital Oakland Research Institute, 5700 Martin Luther King, Jr., Way, Oakland, California 94609, USA. Phone: (510) 450-7908; Fax: (510) 450-7909; E-mail: rmkrauss@lbl.gov.

1. Woollett, L.A., Spady, D.K., and Dietschy, J.M. 1992. Saturated and unsaturated fatty acids independently regulate low density lipoprotein receptor activity and production rate. J. Lipid Res. 33:77-88.

2. Kurushima, H., et al. 1995. Opposite effects on cholesterol metabolism and their mechanisms induced by dietary oleic acid and palmitic acid in hamsters. Biochim. Biophys. Acta. 1258:251-256.

3. Fisher, E.A., and Ginsberg, H.N. 2002. Complexity in the secretory pathway: the assembly and secretion of apolipoprotein B-containing lipoproteins. J. Biol. Chem. 277:17377-17380.

4. Fisher, E.A., et al. 2001. The triple threat to nascent apolipoprotein B. Evidence for multiple, distinct degradative pathways. J. Biol. Chem. 276:27855-27863.

5. Pan, M., et al. 2004. Lipid peroxidation and oxidant stress regulate hepatic apolipoprotein B degradation and VLDL production. J. Clin. Invest. 113:1277-1287. doi: $10.1172 / 200419197$.

6. Kris-Etherton, P.M., Harris, W.S., and Appel, L.J.
2003. Fish consumption, fish oil, omega-3 fatty acids, and cardiovascular disease. Arterioscler. Thromb. Vasc. Biol. 23:e20-e30.

7. Djousse, L., et al. 2003. Dietary linolenic acid is inversely associated with plasma triacylglycerol: the National Heart, Lung, and Blood Institute Family Heart Study. Am. J. Clin. Nutr. 78:1098-1102.

8. Mensink, R.P., Zock, P.L., Kester, A.D., and Katan, M.B. 2003. Effects of dietary fatty acids and carbohydrates on the ratio of serum total to HDL cholesterol and on serum lipids and lipoproteins: a meta-analysis of 60 controlled trials. Am. J. Clin. Nutr. 77:1146-1155.

9. Kummrow, E., Hussain, M.M., Pan, M., Marsh, J.B., and Fisher, E.A. 2002. Myristic acid increases dense lipoprotein secretion by inhibiting apoB degradation and triglyceride recruitment. J. Lipid Res. 43:2155-2163.

10. Berneis, K.K., and Krauss, R.M. 2002. Metabolic origins and clinical significance of LDL heterogeneity. J. Lipid Res. 43:1363-1379.

11. Dreon, D.M., et al. 1998. Change in dietary saturated fat intake is correlated with change in mass of large low-density-lipoprotein particles in men. Am. J. Clin. Nutr. 67:828-836.

12. Krauss, R.M., Lindgren, F.T., and Ray, R.M. 1980. Interrelationships among subgroups of serum lipoproteins in normal human subjects. Clin. Chim. Acta. 104:275-290.

13. Packard, C.J., et al. 2000. Apolipoprotein B metabolism and the distribution of VLDL and LDL subfractions. J. Lipid Res. 41:305-318.

14. Calabresi, L., Donati, D., Pazzucconi, F., Sirtori, C.R., and Franceschini, G. 2000. Omacor in familial combined hyperlipidemia: effects on lipids and low density lipoprotein subclasses. Atherosclerosis. 148:387-396.

15. Heart Protection Study Collaborative Group. 2002. MRC/BHF Heart Protection Study of antioxidant vitamin supplementation in 20,536 high-risk individuals: a randomised placebo-controlled trial. Lancet. 360:23-33.

16. Brown, B.G., et al. 2001. Simvastatin and niacin, antioxidant vitamins, or the combination for the prevention of coronary disease. N. Engl. J. Med. $345: 1583-1592$

\title{
Dysbindin-1 and schizophrenia: from genetics to neuropathology
}

\author{
Michael J. Owen, Nigel M. Williams, and Michael C. O'Donovan
}

Department of Psychological Medicine, University of Wales College of Medicine, Cardiff, United Kingdom.

\begin{abstract}
The gene encoding dysbindin-1 has recently been implicated in susceptibility to schizophrenia. In this issue of the JCI, Talbot et al. show that, contrary to expectations, dysbindin-1 is located presynaptically in glutamatergic neurons and is reduced at these locations in schizophrenia (see the related article beginning on page 1353). Further studies of dysbindin-1 and the proteins with which it interacts can be expected to throw light on the pathogenesis of schizophrenia.
\end{abstract}

Nonstandard abbreviations used: hippocampal formation (HF)

Conflict of interest: The authors have declared that no conflict of interest exists.

Citation for this article: J. Clin. Invest. 113:1255-1257 (2004). doi:10.1172/JCI200421470.
Schizophrenia is a common, severely disabling, mental disorder (1), and understanding its etiology and pathogenesis is one of the most important challenges facing psychiatry. Despite great endeavor, achieving this has proven difficult given the absence of a diagnostic neuropathology or biological markers, and few clear insights have emerged. However, there is currently a consensus that the disorder is, at least in part, neurodevelopmental (2). At the structural level, there are reductions in the neuropil and neuronal size that are widespread but not uniform, with temporal lobe structures, notably the hippocampal formation (HF), particularly affected (3). These changes in turn probably result from alterations in synaptic, dendritic, and axonal organization (3). At the functional level, accumulating evidence also implicates altered glutamate 


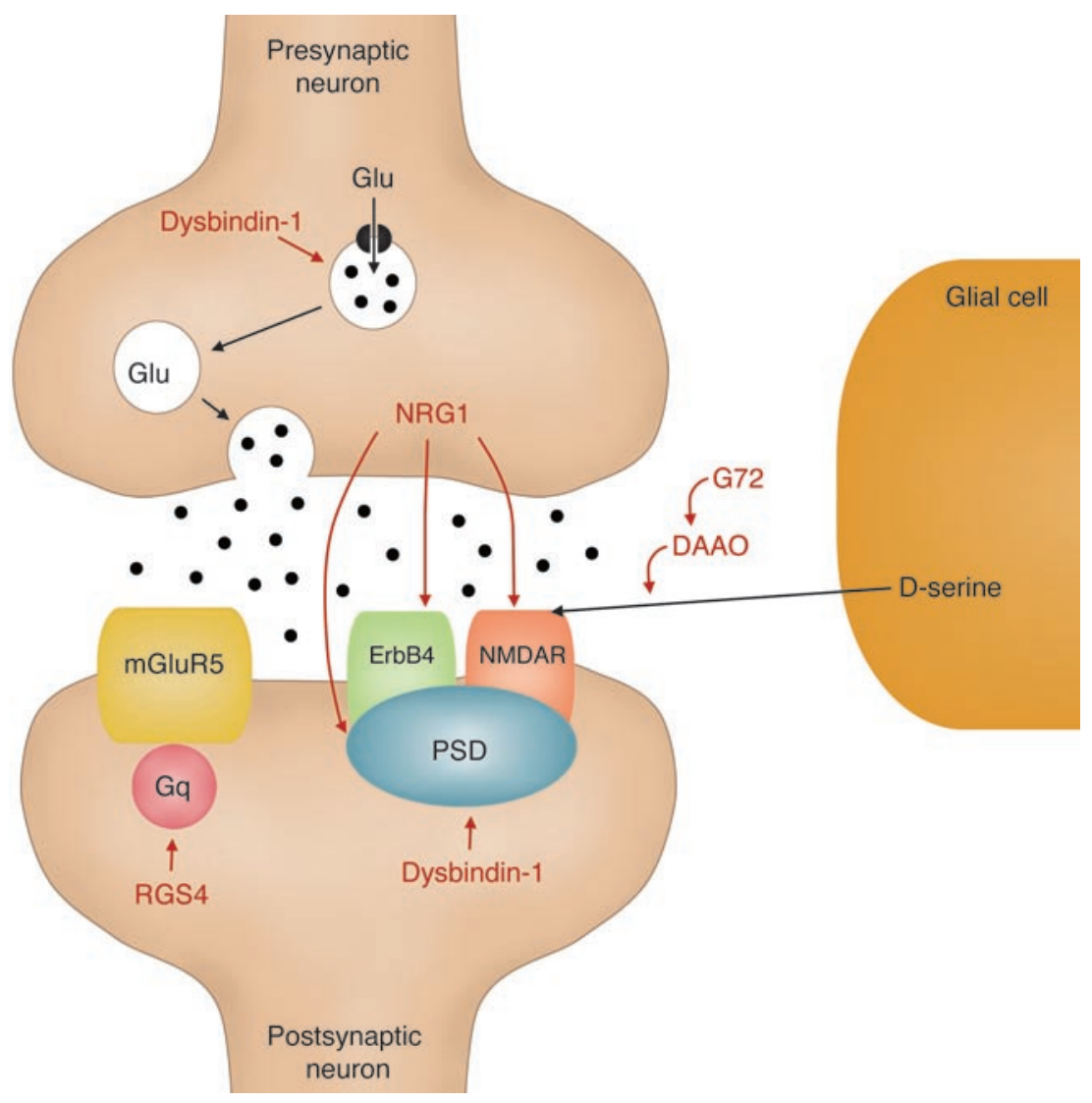

Figure 1

Schematic representation of a glutamatergic synapse. Several genes have been implicated in susceptibility to schizophrenia that can potentially impact on glutamate (Glu) synaptic function including dysbindin-1, neuregulin 1 (NRG1), G72, D-amino acid oxidase (DAAO), and regulator of $\mathrm{G}$ protein signalling 4 (RGS4). Dysbindin-1 may influence VGlutT-1 expression, synthesis, or degradation and is a component of the postsynaptic density (PSD). NRG1 is present in glutamate synaptic vesicles, regulates expression of $N$-methyl-D-aspartate receptors (NMDARs), activates ErbB4 receptors, which colocalize with NMDARs, and interacts with the PSD. G72 interacts with DAAO, which oxidizes D-serine, an endogenous modulator of NMDARs. RGS4 is a negative regulator of $G$ protein-coupled receptors, especially the metabotropic glutamate receptor 5 (mGluR5), via its effects on the G protein Gq. Figure modified with permission from The Lancet (7).

neurotransmission in addition to classical hyperdopaminergic explanations (4).

The most robust body of evidence regarding etiology comes from genetic epidemiological studies, which show that individual differences in liability are predominantly genetic, with heritability estimates around $80 \%$ (5). The most common mode of transmission is probably oligogenic, polygenic, or a mixture of the two (5), but the number of loci, the disease risk conferred by each, the extent of genetic heterogeneity, and the degree of interaction among loci are unknown. As for other common genetic disorders, the small effect on susceptibility conferred by any given locus makes identifying susceptibility genes by positional genetics difficult. However, as sample sizes and hence power have increased, convincing linkages to a number of chromosomal regions have emerged (6). Moreover, systematic examinations of several of these linkage regions have produced replicated evidence implicating specific schizophrenia susceptibility genes $(6,7)$.

\section{Evidence that DTNBP1 is a} susceptibility gene for schizophrenia Currently, the best-supported susceptibility gene is $D T N B P 1$ (e.g., refs. 8-10), which encodes dysbindin- 1 and is located within chromosome 6p22.3. Dysbindin-1 is a $40-50$ $\mathrm{kDa}$ protein that binds both $\alpha$-and $\beta$-dystrobrevin, which are components of the dystro- phin glycoprotein complex (11). The dystrophin complex is found in the sarcolemma of muscle (11) but is also located in postsynaptic densities in a number of brain areas (12). Although its functions are largely unknown, its location initially suggested that genetic variation in DTNBP1 might confer risk of schizophrenia by mediating effects on postsynaptic structure and function (8).

Despite the strong evidence implicating $D T N B P 1$, in the absence of schizophreniaassociated changes that alter the amino acid sequence of dysbindin-1, the actual susceptibility variants remain unknown. It even remains formally possible that these lie within an adjacent gene, but it is more likely that variation within $D T N B P 1$ affects mRNA expression or processing. The latter possibility is indirectly supported by evidence for as yet unknown cis-acting polymorphisms affecting DTNBP1 expression in human brain (13).

\section{Presynaptic reductions of dysbindin-1 in schizophrenia}

In this issue of the JCI, Talbot and colleagues have tested this hypothesis by examining dysbindin-1 protein levels in human brain (14). Their findings provide several novel and important insights. First, while they confirmed fairly widespread neuronal distribution of dysbindin-1 and $\beta$-dystrobrevin, they also showed presynaptic localization of dysbindin-1 but not $\beta$-dystrobrevin, suggesting that dysbindin-1 plays a presynaptic role in the HF that is independent of $\beta$-dystrobrevin and the dystrophin glycoprotein complex. Second, they found high levels of dysbindin-1 in the cells providing the intrinsic glutamatergic pathways of the HF and an inverse correlation with VGlutT-1, the main vesicular glutamate transporter present in the HF. This supports a relationship between dysbindin-1 and glutamate neurotransmission and suggests an effect on VGlutT-1 expression, synthesis, or degradation in the HF. Third, they found a significant reduction in dysbindin-1 expression at these hippocampal presynaptic sites in two separate populations of patients with schizophrenia. If correct, these findings suggest that, in the HF at least, dysbindin-1 may influence schizophrenia risk through presynaptic mechanisms that are independent of the dystrophin glycoprotein complex.

The authors have tried hard, by careful matching of cases and controls, to minimize the potential impact of many of the possible confounding variables that bedevil 
studies of postmortem brain. Most patients with schizophrenia have taken psychotropic medication for many years, and postmortem material from drug-naive patients is extremely difficult to obtain. Talbot and colleagues showed that dysbindin-1 and VGlutT-1 levels were not correlated with antipsychotic drug dose in the month before death and provide evidence against an effect of chronic drug treatment from studies of chronic haloperidol administration to mice (14). However, as the authors acknowledge, the validity of drug-treated mice as a control is open to question, and also no mention is made of matching of cases and controls for agonal state. Given the difficulties confirming previous postmortem findings in schizophrenia, it is now imperative that multiple independent replications be obtained. From the genetic perspective, it would also strengthen the mechanistic case of Talbot and colleagues if disease-associated single nucleotide polymorphisms or haplotypes can be linked to effects on gene expression. Here, ethnic variation in risk haplotypes and possible allelic heterogeneity within populations might prove problematic.

\section{Dysbindin-1 and the pathogenesis of schizophrenia}

These findings raise a number of important issues. First, what is the presynaptic function of dysbindin-1? A possible clue comes from recent evidence implicating it as a member of a protein complex involved in the trafficking of lysosomerelated organelles in association with the proteins muted, pallidin, and cappuccino (15). As Talbot et al. point out, lysosomes are found presynaptically in the $\mathrm{HF}$, and reduced lysosomal trafficking might result in elevated VGlutT-1 (14). However, further studies are required to identify dysbindin-1 interactors and to understand the control of DTNBP1 expression in brain. In addition, analysis of the mouse mutant sandy $(s d y)$, which, in its homozygous form, expresses no dysbindin-1 protein due to a large deletion in Dtnbp1, and of conditional knockouts might also be expected to throw light upon the function of dysbindin- 1 in brain. Second, Talbot and colleagues studied a restricted number of brain regions, particularly the HF. Dysbindin-1 is widely expressed in brain (11), and many lines of evidence suggest widespread involvement of different brain areas in the structural and functional abnormalities of schizophrenia (3). There is now a need to determine whether reduced dysbindin-1 levels are found in other brain areas and whether the association with glutamate is generalized or specific to the relatively restricted population of hippocampal regions implicated by Talbot and colleagues. Interestingly, Talbot and colleagues report regional variation in the distribution of the two dysbindin isoforms studied: the HF and cerebral cortex contained both 50 and 40 $\mathrm{kDa}$ isoforms while the cerebellar cortex contained only the $50 \mathrm{kDa}$ variant, implying that there are differences in their transcriptional control.

The identification of a potential role for dysbindin- 1 in glutamate transmission is of considerable interest. Evidence for glutamate dysfunction in schizophrenia is accumulating from both basic and clinical research (4). Moreover, a case can be made that other susceptibility genes identified on the basis of positional genetic studies, such as those encoding Neuregulin 1, G72, D-amino acid oxidase, and regulator of $G$ protein signalling 4, may have convergent effects upon glutamate synapses (Figure 1 ), although the links are speculative and several caveats remain (7).

Finally, if these findings are confirmed, they will indicate the potential power of positional genetics to home in on novel mechanisms. There are other linkages in schizophrenia that are as strong as that to $6 \mathrm{p} 22.3$, indicating the location of other susceptibility genes (6). On the basis of Talbot and colleagues' work (14) we can expect their identification to yield further crucial advances.
Address correspondence to: Michael J. Owen, Department of Psychological Medicine, Henry Wellcome Building, University of Wales College of Medicine, Heath Park, Cardiff CF14 4XN, United Kingdom. Phone: 44-920 7432 48; Fax: 44-920 7465 54; E-mail: owenmj@cf.ac.uk.

1. Gottesman, I.I. 1991. Schizophrenia genesis: the origins of madness. Freeman. New York, New York, USA. 296 pp.

2. Weinberger, D.R. 1995. From neuropathology to neurodevelopment. Lancet. 346:552-557.

3. Harrison, P.J. 1999. The neuropathology of schizophrenia. A critical review of the data and their interpretation. Brain. 122:593-624.

4. Moghaddam, B. 2003. Bringing order to the glutamate chaos in schizophrenia. Neuron. 40:881-884.

5. Owen, M.J., O’Donovan, M.C., and Gottesman, I.I. 2002. Schizophrenia. In Psychiatric genetics and genomics. P. McGuffin, M.J. Owen, and I.I. Gottesman, editors. Oxford University Press. New York, New York, USA. 247-266.

6. O'Donovan, M.C., Williams, N.M., and Owen, M.J. 2003. Recent advances in the genetics of schizophrenia. Hum. Mol. Genet. 12:R125-R133.

7. Harrison, P.J., and Owen, M.J. 2003. Genes for schizophrenia? Recent findings and their pathological implications. Lancet. 361:417-419.

8. Straub, R.E., et al. 2002. Genetic variation in the 6p22.3 gene DTNBP1, the human ortholog of the mouse dysbindin gene, is associated with schizophrenia. Am. J. Hum. Genet. 71:337-348.

9. Schwab, S.G., et al. 2003. Support for association of schizophrenia with genetic variation in the $6 \mathrm{p} 22.3$ gene, dysbindin, in sib-pair families with linkage and in an additional sample of triad families. Am. J. Hum. Genet. 72:185-190.

10. Williams, N.M., et al. 2004. Identification in two independent samples of a novel schizophrenia risk haplotype of the dystrobrevin binding protein gene (DTNBP1). Arch. Gen. Psychiatry. 61:336-344.

11. Benson, M.A., Newey, S.E., Martin-Rendon, E., Hawkes, R., and Blake, D.J. 2001. Dysbindin, a novel coiled-coil-containing protein that interacts with the dystrobrevins in muscle and brain. J. Biol. Chem. 276:24232-24241.

12. Blake, D.J., Hawkes, R., Benson, M.A., and Beesley, P.W. 1999. Different dystrophin-like complexes are expressed in neurons and glia. J. Cell Biol. 147:645-657

13. Bray, N.J., Buckland, P.R., Owen, M.J., and O'Donovan, M.C. 2003. Cis-acting variation in the expression of a high proportion of genes in human brain. Human Genet. 113:149-153.

14. Talbot, K., et al. 2004. Dysbindin-1 is reduced in intrinsic, glutamatergic terminals of the hippocampal formation in schizophrenia. J. Clin. Invest. 113:1353-1363. doi:10.1172/JCI200420425.

15. Li, W., et al. 2003. Hermansky-Pudlak syndrome type 7 (HPS-7) results from mutant dysbindin, a member of the biogenesis of lysosome-related organelles complex 1 (BLOC-1). Nature Genet. 35:84-89. 